\title{
Editorial
}

\section{Dysphagia - Finally getting the Attention It needed}

Gone are the days when a patient with carcinoma cheek will undergo radical excision and go home with a yawning gap in the operated site. The corrective surgery was not undertaken till the surgeon was convinced that the patient was cured of the disease. The outcome was a simple prolongation of life with severely compromised quality of life. Even when the defect was closed surgically, the thought of making the patient eat and drink normally took a backseat. Similarly, many neurologically damaged patients, either from the disease itself or by the surgical treatment, were never to enjoy the pleasures of enjoying their food. Vast majority of them were dependent on nasogastric tube feeding and few on gastrostomy. The

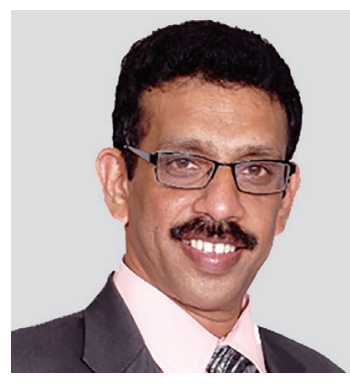

Jayakumar R Menon latter always was an open procedure, many times necessitating general anesthesia and hospitalization. Compounding the problem was the lack of qualified nutritionists and dietitians.

When a patient with a swallowing problem, on nasogastric tube feeding, was examined in the ear, nose, and throat (ENT) outpatient department, the only diagnostic test to be carried out was watching the act of swallowing, apart from a clinical examination of the ENT and the central nervous system. Consequently, it was of little wonder that the ENT surgeons were able to contribute anything substantial. Speech pathologists were not at all involved in the treatment of dysphagia. Though barium swallow was in the vogue since the 1950s, its use in swallowing disorders was restricted to finding any static pathology.

All these things started changing in the last three and a half decades. Langmore emphasized the role of flexible laryngoscope for assessing pharyngeal dysphagia. The dynamic barium swallow became the gold standard for evaluating dysphagia in all the stages. Speech and language pathologists became more interested and proactive in the management of dysphagia patients. Percutaneous endoscopic gastrostomy became a simple procedure performed under local anesthesia. The ENT surgeons became more adept at transnasal esophagoscopy, again reducing the discomfort to the patient and cutting down the cost. Nutritional status of the patients was also addressed, as subjective global assessment scoring system was adapted. Manometric and $\mathrm{pH}$ studies added new horizons to the diagnostic capabilities of the deglutologists.

Phonosurgical techniques like medialization thyroplasty and arytenoid adduction were already in use. They were incorporated into the spectrum of surgical procedures used to treat aspiration. Percutaneous injection laryngoplasty became a very effective office procedure to medialize the paralyzed cord, thus reducing the severity of aspiration. Cricopharyngeal myotomy was already existent for more than 60 years and was sort of reinvented. Botulinum toxin injection to treat muscular dystonias became more and more popular and its use in cricopharyngeal dysmotility was only a natural extrapolation. Resonance correction surgeries like sphincter pharyngoplasty and sleep apnea surgeries like hyoidopexy also found themselves roles in the spectrum of "phagosurgery."

\section{CAREGIVERS}

Of late, there has been tremendous increase in the awareness of dysphagia. Laryngologists, gastroenterologists, neurologists and neurosurgeons, head and neck surgeons, swallowing pathologists, nutritionists, and dietitians all contribute to the cause. Consequently, dysphagia understanding is improving by leaps and bounds. This, no doubt, will help in planning better treatment options and far more favorable outcomes. The patients themselves are more aware of the possible treatment options and hence search for the remedies from the centers specialized in dysphagia management. All these factors make dysphagia management a very challenging yet rewarding field, and it is no surprise to find more and more youngsters in otolaryngology and speech and voice pathology becoming specialists in swallowing disorders management.

\section{EQUIPMENTS}

The challenges facing a physician about to start a swallowing specialty clinic may look formidable at the outset. The extremely expensive gadgets may scare off the aspiring young otolaryngologist. But this problem can be solved by starting with the basic minimum instrumentation and then gradually adding more expensive equipment as the patient load increases. The basic instrument to kick start the program would be a flexible rhinolaryngoscope with 
suction channel. As there is a paradigm shift toward flexible endoscopic evaluation of swallowing (FEES) as the choice investigation in dysphagia, it is certainly worth to start the department with a good flexible laryngoscope. Fluoroscopy is the other basic necessity to start a swallowing unit. A high-end fluoroscope can be very expensive, costing up to Rs 1.5 crore. But less expensive models are available. If shared among other specialties, fluoroscope can be very cost-effective. In case there is no fluoroscope available, borrowing the $\mathrm{C}$-arm from the orthopedic colleagues will be a worthwhile option. A recording facility for both the laryngoscopic and fluoroscopic evaluation is a must, as many subtle findings may be missed at the first viewing. A portable pulse oximeter is a very economical tool for bedside evaluation of swallowing.

All the other equipments can be acquired gradually, thus avoiding the economic burden on the hospital. A transnasal esophagoscope will be a valuable addition to the swallowing unit, as it not only helps in diagnosing the esophageal pathology, but also carries out many therapeutic procedures like removal of foreign bodies, taking biopsies, doing dilatations, and injections. Manometry and pH studies can be added as the number of patients attending the clinic increases. If there is a nuclear medicine department in the hospital, a scintigraphy will be an additional facility that can be used in certain selected cases like microaspiration.

\section{PATIENTS}

The vast majority of patients coming to the dysphagia clinic will be from three groups. Head and neck cancer patients, either due to their illness or due to the treatment modalities often will have significant swallowing disabilities. The second major group will be patients from the neurology and neurosurgical services. Both these groups will have significant swallowing morbidity. An equally large group will be the so-called "globus" patients. Vast majority of them come because of vague symptoms and anxiety. But they also require thorough evaluation of swallowing mechanism, as it is well known that a small percentage of these patients do have structural or functional abnormalities of the swallowing apparatus. In addition to these major groups, referrals can come from geriatric, rheumatology, and dermatology departments. Pediatric dysphagia cases are a real challenge and need very extensive clinical evaluation.

\section{PROTOCOL}

Dysphagia management is evolving daily, thanks to the untiring efforts of the pioneers in the field. As the understanding of the physiology gets better and better, the management options also change. Hence, it is a highly dynamic field of medicine. The physicians and the swallowing pathologists involved should be well versed with the latest development in this ever-changing arena. Setting up a management protocol will certainly give uniformity to the treatment options in various disease conditions. It will also be useful in defending against medicolegal cases as well as for teaching purpose. Needless to say, to continue with the research and compare the results, a standard protocol is a must.

In conclusion, it can be safely ascertained that dysphagia is certainly getting the attention it deserved all these years. As laryngologists, we are in the forefront in this battle to conquer dysphagia. While we may not be able to make all these unfortunate patients eat a three-course dinner, we can certainly make majority of them get rid of their feeding tubes. Even in those patients, who continue to be feeding tube dependent, the dysphagia team may be able to make many of them eat at least something. Even in the most refractory dysphagia patients, encouragement and sympathetic attitude may sometimes work wonders.

Jayakumar R Menon

Senior Consultant

Department of Laryngology, Kerala Institute of Medical Sciences Thiruvananthapuram, Kerala, India 